07

\title{
Применение неохлаждаемых микроболометров для регистрации импульсного терагерцового и инфракрасного излучения
}

\author{
(C) М.A. Демьяненко, ${ }^{1}$ В.В. Старцев ${ }^{2}$ \\ ${ }^{1}$ Институт фоизики полупроводников им. А.В. Ржанова СО РАН, \\ 630090 Новосибирск, Россия \\ ${ }^{2}$ Акционерное общество „Оптико-механическое конструкторское бюро Астрон“, \\ 140080 Лыткарино, Московская обл., Россия \\ e-mail: demyanenko@isp.nsc.ru
}

Поступило в Редакцию 21 июня 2021 г.

В окончательной редакции 17 ноября 2021 г.

Принято к публикации 26 ноября 2021 г.

Получены аналитические соотношения для температурного отклика болометра на периодические импульсы излучения. Теоретически показано и на примере инфракрасных болометров экспериментально подтверждено, что при регистрации коротких импульсов излучения в отличие от случая постоянного излучения, повышая теплопроводность болометра и соответственно понижая время его тепловой релаксации, можно существенно повысить быстродействие приемника, практически не снижая его чувствительности. Рассмотрена возможность эффективной регистрации терагерцового импульсного излучения микроболометрами с резистивно-связанной, термически неизолированной антенной. Показано, что такие болометры, обладающие повышенной теплопроводностью и соответственно пониженной чувствительностью к излучению постоянной интенсивности, могут быть высокоэффективными при регистрации импульсов излучения длительностью меньше времени тепловой релаксации болометра. На их основе могут быть разработаны неохлаждаемые матричные приемники импульсного терагерцового излучения, характеризующиеся минимальной обнаружимой энергией менее $1 \cdot 10^{-12} \mathrm{~J}$ и кадровой частотой до $1000 \mathrm{~Hz}$.

Ключевые слова: микроболометр, импульсное терагерцовое излучение, антенна.

DOI: 10.21883/JTF.2022.03.52139.190-21

\section{Введение}

Матричные неохлаждаемые микроболометрические приемники традиционно применяются в системах регистрации инфракрасного (ИК) [1,2] и терагерцового (THz) излучения [3-9] с постоянной или медленно меняющейся интенсивностью, т. е. когда характерное время изменения падающей на приемник мощности излучения значительно больше времени тепловой релаксации болометров. Однако в ряде случаев применение импульсного излучения позволяет повысить точность измерения вследствие устранения постоянных или низкочастотных паразитных сигналов. В ряде задач, например при необходимости регистрации THz-излучения, отраженного от удаленных объектов, требуется подсветка мощным излучением. В таких случаях для понижения энергетических затрат и повышения безопасности применяется импульсное излучение малой длительности, подобно тому как это делается при дистанционном зондировании атмосферы $[10,11]$. Кроме того, некоторые источники THz-излучения, например, основанные на фотопроводящих антеннах $[12,13]$ и на нелинейных оптических кристаллах [14], имеют импульсный характер работы, или, подобно квантовым каскадным лазерам [15-17], обладают значительно большей мощностью в импульсном режиме работы. Применение неохлаждаемых микроболометрических приемников для регистрации импульс- ных сигналов, с одной стороны, требует проведения дополнительного анализа особенностей их работы, а с другой стороны, приводит к необходимости пересмотра конструктивных подходов, применяемых при разработке самих приемников с целью достижения высокой чувствительности к импульсному излучению. В первую очередь это касается приемников THz-диапазона, в которых высокая чувствительность обеспечивается применением, во-первых, антенн, резистивным или емкостным способом связанных с нагрузкой, расположенной на термоизолированной мембране болометра $[8-9,18]$, и, вовторых, наносимых на мембрану болометра поглотителей излучения, основанных на тонких металлических пленках [5,7] и метаматериалах [19]. Приемники с антеннами можно разделить на два типа. Первый - болометры, сочетающие применение антенны, расположенной на подложке и сопряженной с нагрузкой емкостной связью, и резистивно-связанной антенны, расположенной на термоизолированной мембране болометра [8-9]. Эти приемники обладают малой теплопроводностью. Второй - болометры с резистивно-связанной, термически неизолированной (расположенной на подложке) антенной [18]. Такие приемники обладают повышенной теплопроводностью, обусловленной шиной резистивной связи между антенной и нагрузкой. Это делает их менее чувствительными к постоянному THz-излучению, но при этом более быстродействующими вследствие 
меньшего времени тепловой релаксации. При регистрации импульсного излучения влияние теплопроводности на величину сигнала значительно ослабляется, и такие приемники могут оказаться высокоэффективными.

В настоящей работе теоретически и экспериментально исследуются зависимости величины сигнала болометра от параметров импульса ИК или THz-излучения и времени тепловой релаксации болометра. Рассматриваются основные конструктивные особенности изготовления неохлаждаемых матричных приемников на основе микроболометров с резистивно-связанной, термически неизолированной антенной, предназначенных для эффективной регистрации THz-импульсного излучения.

\section{1. Температурный отклик болометра при импульсном освещении}

Уравнение теплового баланса болометра при воздействии на него поглощенного излучения сигнала, зависящего от времени $t$, и постоянного фона, мощности которых соответственно равны $P_{S}(t)$ и $P_{B}$, имеет вид

$$
C \frac{d T_{D}}{d t}=P_{S}(t)+P_{B}+Q_{D}\left(T_{D}\right)-G_{0}\left(T_{D}-T_{C}\right) .
$$

Здесь $T_{D}$ и $T_{C}$ - температура болометра и подложки, на которой он изготовлен; $C$ и $G_{0}-$ теплоемкость и теплопроводность болометра, которые ввиду малой разницы $T_{D}$ и $T_{C}$ будем считать зависящими только от температуры $T_{C} ; Q_{D}$ - мощность джоулевого тепла, выделяющегося в болометре при протекании в нем тока. Предполагается, что теплоемкость и теплопроводность маленьких теплоизолирующих балок много меньше теплоемкости и теплопроводности мембраны болометра, что обеспечивает однородность ее температуры, которую будем считать температурой болометра $T_{D}$. Под воздействием излучения сигнала изменяются сопротивление болометра $R_{D}$ и соответственно $Q_{D}$, что приводит к дополнительному изменению температуры $T_{D}$ в результате так называемого термоэлектрического взаимодействия или термоэлектрической обратной связи $[20]$. В случае включения болометра последовательно с источником постоянного напряжения $V_{0}$ и нагрузочным сопротивлением, величина которого $R_{L}$ не зависит от выделяющейся в нем мощности, изменение мощности выделяющегося джоулевого тепла $Q_{D}$ описывается соотношением $d Q_{D}\left(T_{D}\right)=\beta \alpha Q_{D O} d T_{D} \quad[20,21]$. Здесь $\alpha=\left(d R_{D} / d T_{D}\right) / R_{D}$ - температурный коэффициент сопротивления (ТКС), $\beta=\left(R_{L}-R_{D O}\right) /\left(R_{L}+R_{D O}\right)$, $R_{D O}$ и $Q_{D O}$ - значения $R_{D}$ и $Q_{D}$ при рабочей температуре болометра $T_{O}$, определяемой соотношением $T_{O}=T_{C}+\left(P_{B}+Q_{D O}\right) / G_{0}$. Раскладывая $Q_{D}\left(T_{D}\right)$ в ряд Тейлора вблизи рабочей точки $T_{O}$ до членов первого порядка, соотношение (1) можно записать в виде [20]:

$$
\frac{d T}{d t}+\frac{T}{\tau}=\frac{P_{S}(t)}{C}
$$

Здесь $T=T_{D}-T_{O}-$ отклик болометра на излучение сигнала, $\tau=C / G$ и $G-$ эффективное время тепловой релаксации болометра и эффективная теплопроводность, заданная соотношением $G=G_{0}-\beta \alpha Q_{D O}=G_{0}\left(1-\beta \alpha T_{Q O}\right), T_{Q O}=Q_{D O} / G_{0}-$ величина разогрева болометра выделяющимся джоулевым теплом в рабочей точке. Величина $\alpha T_{Q O}$ может быть порядка единицы. Действительно, разогрев болометра, сопротивление $R_{D}$, ТКС $\alpha$ и теплопроводность $G_{0}$ которого равны $100 \mathrm{k} \Omega$, — $2 \% / \mathrm{K}$ и $10^{-7} \mathrm{~W} / \mathrm{K}[2,21]$, составит примерно $25 \mathrm{~K}\left(\alpha T_{Q O}=-0.5\right)$ при напряжении смещения болометра $V_{D}=V_{0} R_{D O} /\left(R_{L}+R_{D O}\right)=0.5 \mathrm{~V}$. Отметим, что уравнение (2) позволяет, с одной стороны, по заданной зависимости мощности поглощенного излучения сигнала от времени $P_{S}(t)$ найти временную зависимость температурного отклика болометра $T(t)$, a с другой - по измеренной зависимости $T(t)$ определить зависимость мощности сигнала от времени $P_{S}(t)$. В частности, при постоянной мощности сигнала $P_{S}$ получим $T=P_{S} \tau / C=P_{S} / G$.

Общее решение уравнения (2) имеет вид [22]:

$T(t)=T\left(t_{0}\right) \exp \left(-\frac{t-t_{0}}{\tau}\right)+\int_{t_{0}}^{t} \frac{P_{S}\left(t^{\prime}\right)}{C} \exp \left(-\frac{t-t^{\prime}}{\tau}\right) d t^{\prime}$

где $T\left(t_{0}\right)$ - значение $T$ в некоторый момент времени $t_{0}$. Если излучение имеет периодическую временную зависимость с периодом $\Theta$, то для любого $t_{0}$ выполняется равенство $T\left(t_{0}+\Theta\right)=T\left(t_{0}\right)$. Это с учетом соотношения (3) позволяет получить, что

$$
T\left(t_{0}\right)=\frac{\exp (-\Theta / \tau)}{[1-\exp (-\Theta / \tau)]} \int_{t_{0}}^{t_{0}+\Theta} \frac{P_{S}\left(t^{\prime}\right)}{C} \exp \left(\frac{t^{\prime}-t_{0}}{\tau}\right) d t^{\prime}
$$

В случае импульсного излучения, такого, что $P_{S}(t)$ не равна нулю только в интервале $t_{1}<t<t_{2}\left(t_{1}=t_{S}-t_{p e} / 2\right.$, $t_{2}=t_{S}+t_{p e} / 2, t_{S}$ - момент времени прихода условной середины импульса, $t_{p e}$ - эффективная длительность импульса), соотношения (4) и (3) преобразуются к виду

$$
\begin{gathered}
T\left(t_{1}\right)=D T\left(t_{1}, t_{2}\right) \frac{\exp (-\Theta / \tau)}{[1-\exp (-\Theta / \tau)]} \exp \left(-\frac{t_{1}-t_{S}}{\tau}\right), \\
T(t)=T\left(t_{1}\right) \exp \left(-\frac{t-t_{1}}{\tau}\right)+D T\left(t_{1}, t\right) \exp \left(-\frac{t-t_{S}}{\tau}\right), \\
T(t)=D T\left(t_{1}, t_{2}\right) \frac{\text { при } t_{1}<t<t_{2},}{[1-\exp (-\Theta / \tau)]} \exp \left(-\frac{t-t_{S}}{\tau}\right) . \\
\text { при } t_{2}<t<t_{1}+\Theta
\end{gathered}
$$

где

$$
D T\left(t_{1}, t\right)=\int_{t_{1}}^{t} \frac{P_{S}\left(t^{\prime}\right)}{C} \exp \left(\frac{t^{\prime}-t_{S}}{\tau}\right) d t^{\prime}
$$


Если $t_{p e} \ll \tau$, то, проводя разложение соотношения (7) в ряд по малому параметру $t_{p e} / \tau$ и пренебрегая членами первого и более высокого порядка, найдем, что $D T\left(t_{1}, t\right) \approx Q_{S}(t) / C$ и $D T\left(t_{1}, t_{2}\right) \approx Q_{S} / C$, где $Q_{S}$ и $Q_{S}(t)$ - энергии импульса излучения, поглощенные в течение всей длительности импульса и в интервале времени $t_{1}-t$ соответственно. Для симметричного относительно точки $t_{S}$ импульса излучения указанное разложение $D T\left(t_{1}, t_{2}\right)$ не будет содержать членов первого порядка, и, следовательно, соотношения (5) и (6b), характеризующие температурный отклик $T(t)$ в моменты времени между импульсами, после замены в них $D T\left(t_{1}, t_{2}\right)$ на $Q_{S} / C$ будут выполняться с точностью до членов второго порядка малости по $t_{p e} / \tau$. В этом случае для температурного отклика перед приходом импульса и после его прохождения имеем:

$$
\begin{gathered}
\left.T\left(t_{1}\right) \approx\left(Q_{S} / C\right) \exp \left[-\left(t_{1}-t_{S}\right) / \tau\right)\right] /[\exp (\Theta / \tau)-1], \\
\left.T\left(t_{2}\right) \approx\left(Q_{S} / C\right) \exp \left[-\left(t_{2}-t_{S}\right) / \tau\right)\right] /[1-\exp (-\Theta / \tau)],
\end{gathered}
$$

а его прирост в течение импульса составит

$$
\begin{aligned}
T\left(t_{2}\right)-T\left(t_{1}\right) & \approx\left(Q_{S} / C\right) \operatorname{sh}\left[\left(\Theta-t_{p e}\right) / 2 \tau\right] / \operatorname{sh}(\Theta / 2 \tau) \\
& \approx\left(Q_{S} / C\right)\left[1-\left(t_{p e} / 2 \tau\right) \operatorname{cth}(\Theta / 2 \tau)\right] .
\end{aligned}
$$

Из последнего соотношения видно, что, если $\Theta$ больше или порядка $\tau$ и $t_{p e} \ll \tau$, то $T\left(t_{2}\right)-T\left(t_{1}\right) \approx Q_{S} / C$. Это означает, что вся поглощенная болометром энергия импульса излучения идет на его разогрев, а оттоком тепла в течение импульса за счет теплопроводности болометра можно пренебречь. Если же $\Theta \ll \tau$, то

$$
\begin{gathered}
T\left(t_{1}\right) \approx\left(Q_{S} / C\right)(\tau / \Theta)\left(1+t_{p e} / 2 \tau\right) /(1+\Theta / 2 \tau), \\
T\left(t_{2}\right) \approx\left(Q_{S} / C\right)(\tau / \Theta)\left(1-t_{p e} / 2 \tau\right) /(1-\Theta / 2 \tau)
\end{gathered}
$$

и

$$
T\left(t_{2}\right)-T\left(t_{1}\right) \approx\left(Q_{S} / C\right)\left(1-t_{p e} / \Theta\right) .
$$

В этом случае для того, чтобы прирост температуры болометра в течение импульса составил $Q_{S} / C$, потребуется выполнение более жесткого условия $t_{p e} \ll \Theta \ll \tau$. Это обусловлено тем, что при $\Theta \ll \tau$ перед приходом очередного импульса излучения болометр не успевает остыть после воздействия на него нескольких предыдущих импульсов, облучающих болометр за время его тепловой релаксации $\tau$, количество которых можно оценить величиной $\tau / \Theta$. В результате болометр разогревается до температуры $T\left(t_{1}\right) \sim\left(Q_{S} / C\right)(\tau / \Theta) \gg Q_{S} / C$. В такой ситуации скорость оттока тепла за счет теплопроводности $G$ повышается в $\tau / \Theta$ раз по сравнению со случаем $\Theta>\tau$, когда болометр успевает значительно остыть перед приходом следующего импульса, что и приводит к необходимости понижения продолжительности импульса также в $\tau / \Theta$ раз, для того чтобы отток энергии в течение длительности импульса был много меньше поглощенной энергии импульса излучения. Заметим, что $\left(Q_{S} / C\right)(\tau / \Theta)=\left\langle P_{S}\right\rangle / G$, где $\left\langle P_{S}\right\rangle=Q_{S} / \Theta-$ мощность

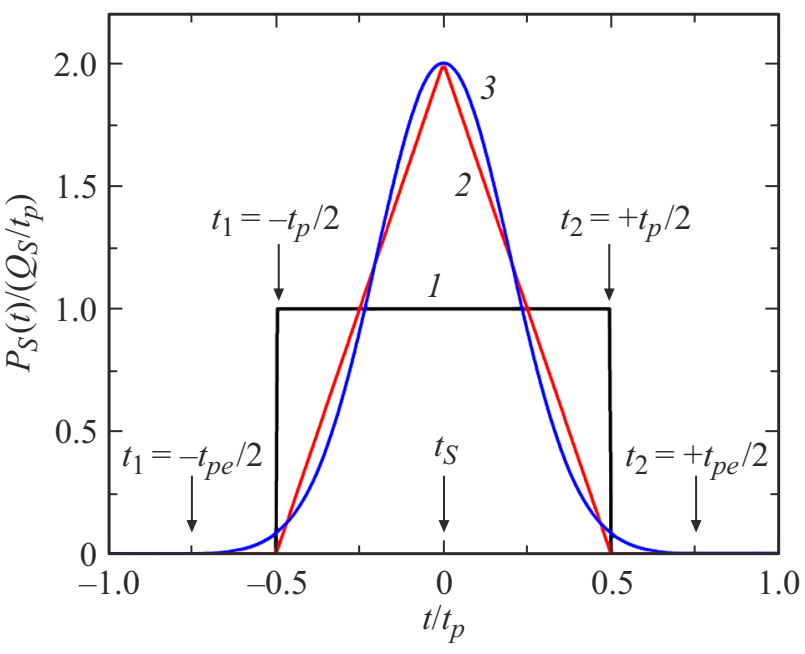

Рис. 1. Нормированные зависимости мощности поглощенного болометром излучения $P_{S}(t)$ от времени $t$ для импульсов излучения различной формы: 1 - прямоугольная, 2 - треугольная, 3 - гауссова, используемых при расчете температурного отклика болометра, показанного на рис. 2; $t_{S}$ - момент времени прихода середины импульса, $t_{p}-$ параметр, задающий длительность импульса, $t_{1}$ и $t_{2}-$ задают интервал времени, вне которого $P_{S}(t)$ считается равной нулю, $t_{p e}=t_{2}-t_{1}-$ эффективная длительность импульса.

излучения, усредненная за период, и, следовательно, в нулевом приближении по $t_{p e} / \tau$ и $\Theta / \tau$ находим, что $T\left(t_{1}\right) \approx T\left(t_{2}\right) \approx\left\langle P_{S}\right\rangle / G$

В общем случае, когда эффективная длительность импульса излучения $t_{p e}$ может быть сравнима со временем тепловой релаксации $\tau$, отток тепла в течение импульса излучения за счет теплопроводности $G$ может стать значительным даже при $\Theta \gg \tau$ и для расчета величин $D T\left(t_{1}, t\right)$ и $D T\left(t_{1}, t_{2}\right)$ необходимо использовать интегральное выражение (7).

В качестве иллюстрации сказанного выше рассмотрим зависимости температурного отклика болометра от времени при воздействии на него трех типов импульсного излучения (рис. 1), обладающих одинаковой полной энергией $Q_{S}$ и симметричных относительно момента времени прихода их середины $t_{S}$, принятого равным нулю:

1) прямоугольный импульс:

$$
P_{S}(t)=Q_{S} / t_{p} \text { при }-t_{p} / 2<t<t_{p} / 2,
$$

2) треугольный импульс:

$$
P_{S}(t)=2\left(Q_{S} / t_{p}\right)\left[1-\left|t /\left(t_{p} / 2\right)\right|\right], \quad-t_{p} / 2<t<t_{p} / 2,
$$

3) гауссов импульс:

$$
P_{S}(t)=\frac{Q_{S}}{\sqrt{2 \pi}\left(t_{p} / b\right)} \exp \left[\frac{-t^{2}}{2\left(t_{p} / b\right)^{2}}\right], \text { где } b=2 \sqrt{2 \pi} \text {. }
$$

Для прямоугольного и треугольного импульсов мощность поглощенного излучения $P_{S}(t)$ не равна нулю 

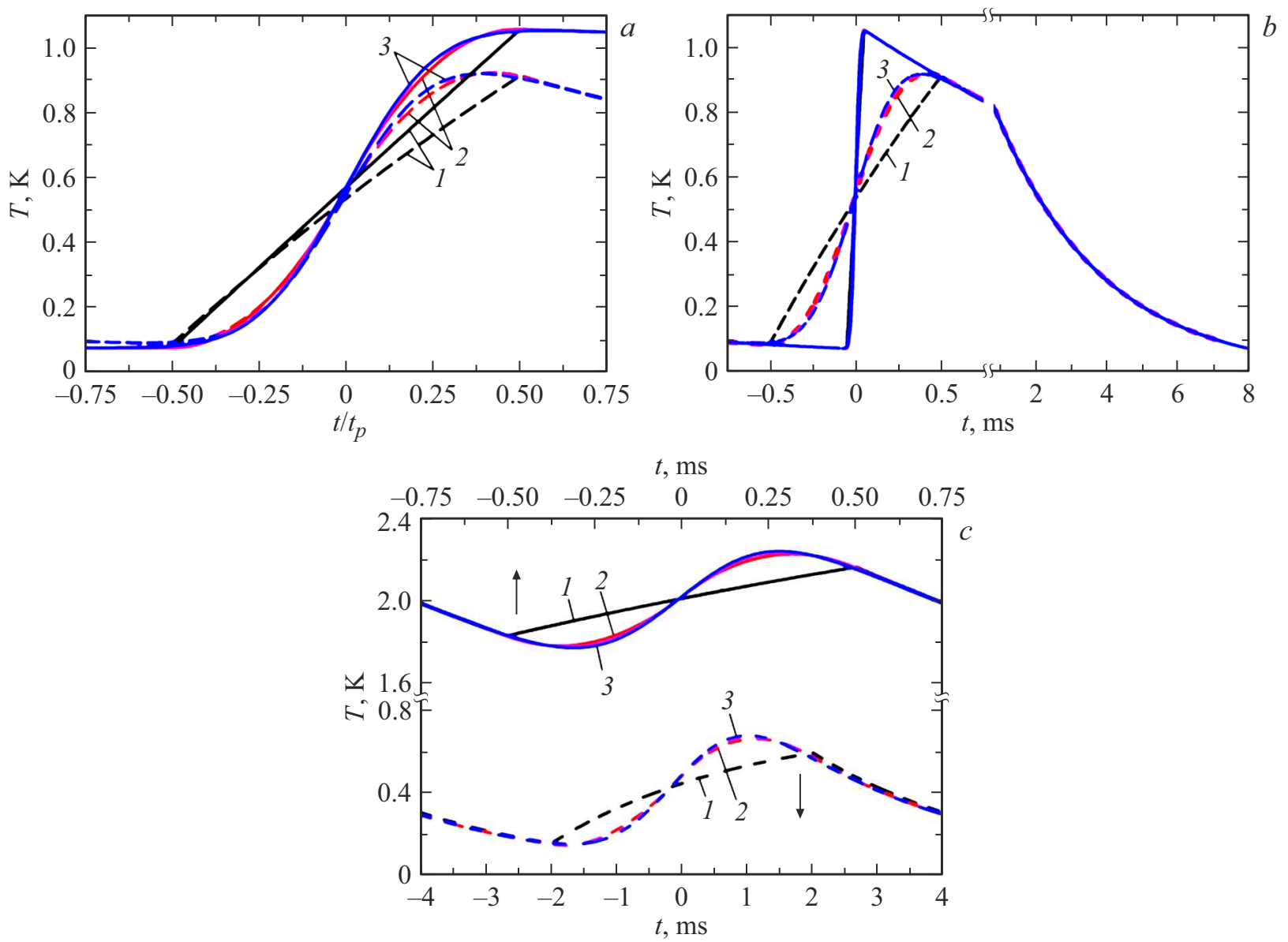

Рис. 2. Зависимости температурного отклика болометра $T$ от времени $t$ под воздействием импульсного излучения различной формы, длительности импульса $t_{p}$ и периода его следования $\Theta: t_{p}=0.1 \mathrm{~ms}$ (сплошная), $t_{p}=1 \mathrm{~ms}($ штриховая), $\Theta=8 \mathrm{~ms}(a, b)$; $t_{p}=1 \mathrm{~ms}$ и $\Theta=1.5 \mathrm{~ms}$ (сплошная), $t_{p}=4 \mathrm{~ms}$ и $\Theta=8 \mathrm{~ms}$ (штриховая) (c). Форма импульса на всех рисунках указана цифрами: 1 - прямоугольная, 2 - треугольная, 3 - гауссова. Поглощенная энергия импульса излучения $Q_{S}=1 \mathrm{~nJ}$, время тепловой релаксации болометра $\tau=3 \mathrm{~ms}$, теплоемкость болометра $C=1 \mathrm{~nJ} / \mathrm{K}$.

только между $t_{1}=-t_{p} / 2$ и $t_{2}=t_{p} / 2$, т.е. в данном случае эффективную длительность импульса $t_{p e}$, за пределами которой мощность падающего излучения $P_{S}(t)$ равна нулю, можно выбрать равной $t_{p}$. В случае гауссова импульса хвосты распределения $P_{S}(t)$ простираются и вне интервала $-t_{p} / 2<t<t_{p} / 2$, поэтому следует выбрать $t_{1}=-n\left(t_{p} / 2\right), t_{2}=n\left(t_{p} / 2\right)$ и $t_{p e}=n t_{p}$, где $n$ равно, например, 1.5. Тогда можно считать, что вне интервала $\left(t_{1}, t_{2}\right) P_{S}(t)$ с большой точностью равна нулю $\left[P_{S}\left(t_{1}\right) / P_{S}(0)=P_{S}\left(t_{2}\right) / P_{S}(0) \approx 8.5 \cdot 10^{-4}\right]$. Отметим, что в случае трех указанных выше импульсов, часто применяемых на практике, удается получить аналитические выражения для $D T\left(t_{1}, t\right)$ и $D T\left(t_{1}, t_{2}\right)$, которые приведены в Приложении и могут быть полезны, например, при необходимости проведения быстрых расчетов или анализа влияния параметров импульсов излучения и самого болометра на величину и форму температурного отклика.

Рассчитанные по соотношениям (5)-(7) зависимости температурного отклика болометра $T$ от времени $t$ при воздействии на него трех вышеуказанных типов импульсного излучения приведены на рис. 2. Из графиков видно, что если $t_{p}$ достаточно мало по сравнению с $\tau$ и $\Theta$, то разность температур сразу после прохождения импульса $T\left(t_{2}\right)$ и непосредственно перед его приходом $T\left(t_{1}\right)$ приблизительно равна $Q_{S} / C$, и точность этого равенства возрастает с уменьшением $t_{p} / \tau$ (рис. $2, a, b$ ). В этом случае отток тепла в подложку на протяжении импульса мал. Если период $\Theta$ заметно превышает $t_{p}$ и $\tau$, то болометр практически успевает остыть к приходу следующего импульса (рис. $2, b$ ). При $\Theta<\tau$ болометр не успевает остыть между импульсами и в соответствии с изложенным выше температурный отклик $T\left(t_{1}\right)$ перед приходом очередного импульса, в значительной мере определяясь средней за период $\Theta$ мощностью излучения, может оказаться существенно выше величины $Q_{S} / C$ (сплошные кривые на рис. 2,c). Это повышает отток тепла в подложку, в результате чего прирост температурного отклика в течение импульса $T\left(t_{2}\right)-T\left(t_{1}\right)$ оказывается значительно меньше $Q_{S} / C$ (при не выпол- 
нении условия $t_{p} \ll \Theta$ ) даже если $t_{p} \ll \tau$ (сплошные кривые на рис. $2, c)$. Важно отметить, что в соответствии с вышеизложенным температурные отклики $T$, измеренные между импульсами излучения при одинаковых значениях времени $t-t_{S}$, слабо зависят от формы и длительности импульсов, если период их следования одинаков и $t_{p} \ll \tau$ (рис. $\left.2, b\right)$. Это справедливо даже в тех случаях, когда отток тепла в течение импульса не является малым (сплошные кривые на рис. 2, с). Заметим также, что для импульсов треугольной и гауссовой форм максимальные значения температуры достигаются до окончания импульса. Это особенно хорошо заметно в случаях, когда отток тепла в подложку на протяжении импульса является существенным (рис. 2, c).

\section{2. Экспериментальное исследование зависимости сигнала болометра от времени тепловой релаксации при импульсном освещении}

Исследовался отклик матричного микроболометрического приемника (ММБП) на импульсное ИК излучение в зависимости от величины времени тепловой релаксации болометра $\tau$, которое изменялось путем изменения давления внутри вакуумного корпуса приемника. Считывание сигналов с элементов ММБП проводилось построчно с частотой кадров $25 \mathrm{~Hz}$, так что измерения элементов каждой следующей строки производилось через $40 \mathrm{~ms} / 241=166 \mu$ s после измерения предыдущей. (В процессе считывания сигналов с ММБП форматом $320 \times 240$ добавлялась одна „служебная“ строка.) Таким образом, однородно освещая некоторый столбец микроболометров импульсным излучением и записывая в файл считываемые с ММБП сигналы текущего кадра, можно измерить временную зависимость реакции микроболометров на импульсное излучение.

В режиме построчного считывания сигналов с ММБП смещение болометров производилось в течение $30 \mu \mathrm{s}$ один раз за кадр (т.е. один раз в течение $40 \mathrm{~ms}$ ). Остальное время ток через болометр не протекает и, следовательно, зависимость температуры болометра от времени будет определяться соотношением (2), в котором $\tau=C / G_{0}$. Влияние термоэлектрической обратной связи происходит только во время приложения импульса смещения болометра. Это приводит к тому, что измеряемый сигнал $V_{S}$ связан с рассчитанным без учета термоэлектрической обратной связи сигналом $V_{S 0}$ следующим соотношением: $V_{S}=V_{S 0} /\left(1-\beta \alpha T_{i 0}\right)$ [23]. Здесь $\Delta T_{i 0}$ - разогрев болометра, обусловленный импульсом смещения, который при напряжении смещения болометpa $V_{D}=2 \mathrm{~V}$ (остальные параметры остаются прежними: $R_{D}=100 \mathrm{k} \Omega, \alpha=-2 \% / \mathrm{K}$ и $\left.C=1 \mathrm{~nJ} / \mathrm{K}\right)$ оказывается равным $1.2 \mathrm{~K}$, так что поправкой $\beta \alpha T_{i 0}$ можно пренебречь. В используемом в эксперименте матричном микроболометрическом приемнике применялась мостовая схема измерения, в которой $R_{D} \approx R_{L}$ и, следовательно,

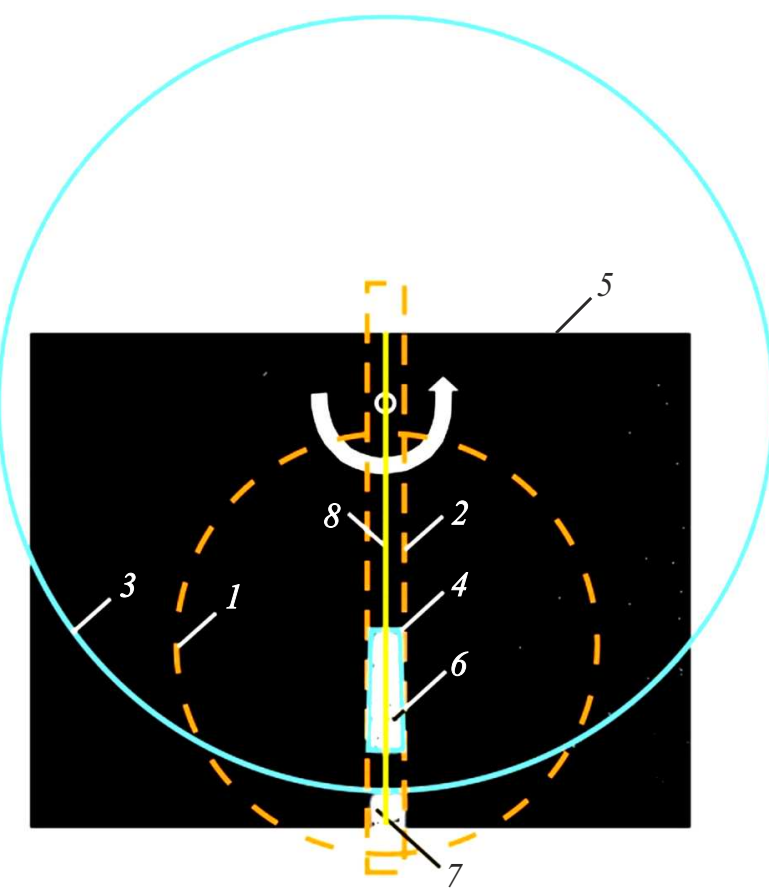

Рис. 3. Схематическое изображение проекций АЧТ (1), щелевой диафрагмы (2), диска модулятора (3) и прорези в нем (4) на чувствительное поле ММБП (5), на котором образуются области, освещенные как импульсным (6), так и постоянным излучением (7). Измерения сигнала производились по столбцу микроболометров, показанному линией $(8)$. Темное поле приемника - нет сигнала, белое - сигнал.

$\beta \approx 0$, что дополнительно уменьшает влияние термоэлектрической обратной связи.

Импульс ИК излучения формировался следующим способом (рис. 3). Эталонный протяженный излучатель типа „абсолютно черное тело“ (АЧТ) марки АЧТ-4И, диаметром $75 \mathrm{~mm} \mathrm{1,} \mathrm{нагретый} \mathrm{до} \mathrm{температуры} 90^{\circ} \mathrm{C}$, помещался за щелевой диафрагмой 2 шириной $8 \mathrm{~mm}$, перед которой вращался диск 3 модулятора с прорезью 4 шириной 6 угловых градусов, расположенной от оси вращения на расстоянии от 60 до $90 \mathrm{~mm}$. Изображение щелевой диафрагмы фокусировалось на ММБП форматом $320 \times 240$ и с размером пикселя $51 \mu \mathrm{m}$, изготовленный в ИФП СО РАН (г.Новосибирск) [24]. При вращении диска на фоточувствительном поле ММБП 5 образовывались две освещенные области. Одна область 6 освещалась импульсами излучения, проходящими через прорезь 4, а вторая 7, соответствующая части щелевой диафрагмы 2, находящейся за краем вращающегося диска 3 , освещалась постоянным (немодулированным) излучением. Медленно поворачивая диск 3 и измеряя сигнал по 171-ому столбцу микроболометров, показанному на рис. 3 линией 8 и соответствующему середине изображения щелевой диафрагмы 2, определяли зависимость мощности падающего ИК излучения от угла поворота диска, и, следовательно, форму импульса излучения, которая была в дальнейшем использована при расчетах. 


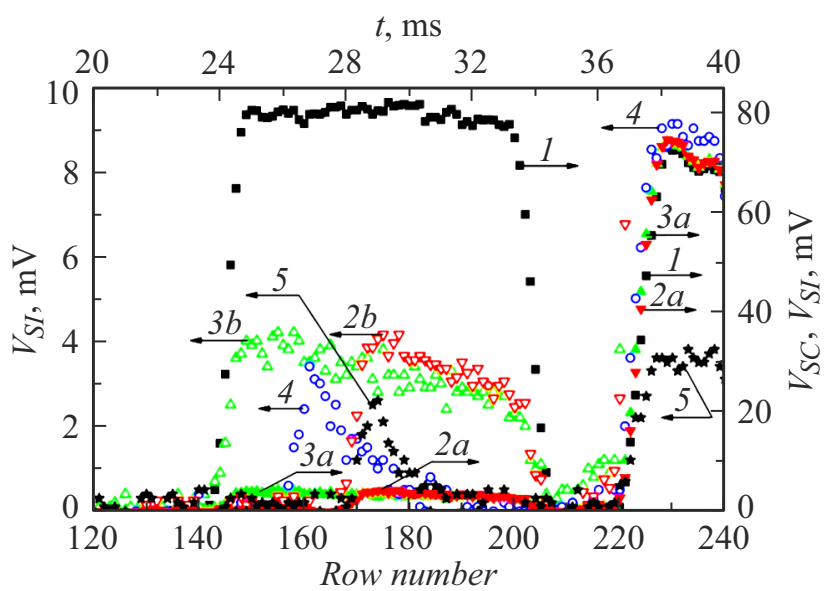

Рис. 4. Распределение величины сигнала по 171-му столбцу ММБП с неподвижным (1) и вращающимся (2-5) диском при высоком $(1-3)$ и низком $(4,5)$ вакууме. Верхняя шкала отражает временную зависимость сигналов в импульсно-освещаемой области (строки 145-205).

Было установлено, что угловая ширина полученного распределения на уровне $10 \%$ в полтора раза превышает геометрическую ширину прорези 4. Так что при вращении диска с частотой $25 \mathrm{~Hz}$ длительность ИК импульса на линии 8 примерно составляла $1.5 \cdot(40 \mathrm{~ms} / 60)=1 \mathrm{~ms}$, что значительно меньше времени тепловой релаксации болометров ММБП при высоком вакууме $\tau_{0}$, которое равно $15 \mathrm{~ms}$.

На рис. 4 приведены распределения величин сигналов вдоль 171-го столбца ММБП (нижняя шкала), измеренные с неподвижным 1 и вращающимся 2-5 диском, при высоком 1-3 и низком 4,5 вакууме. Шкала времени (верхняя шкала) отсчитывается от начала кадра (от „служебной“ строки, расположенной перед первой строкой). Распределение сигналов, измеренное при высоком вакууме и без вращения диска (положение прорези диска совпадает со щелью диафрагмы), является достаточно однородным в области прорези диска (строки 145-205), что соответствует однородному освещению вдоль измеряемого столбца микроболометров. Значения сигнала микроболометров, находящихся в строках от 220 до 240, соответствующих области 7 , показанной на рис. 3, которая всегда освещается постоянным (немодулированным) излучением, немного меньше, возможно, вследствие неоднородности засветки на краю матрицы. Заметим, что, поскольку частота считывания кадров ММБП и частота вращения диска между собой не были синхронизированы, импульс излучения не всегда попадал в интервал времени опроса строк в импульсно освещаемой области (строки 145-205), а в случае попадания - положение начала импульса достаточно случайно. Так, например, при измерении зависимости 2 импульс излучения пришел в момент считывания 170 строки, а при измерении зависимости 3 - примерно в момент считывания 145 строки или немного раныше.

При вращении диска и высоком вакууме величина сигнала в области прорези диска, освещаемой импульсами длительностью $t_{p}=1 \mathrm{~ms}$, существенно меньше сигнала в постоянно освещаемой области 7 , показанной на рис. 3 . Это обусловлено тем, что в соответствии с изложенным в разд. 1 сигнал болометра $V_{S I}$ при его освещении импульсами длительностью $t_{p} \ll \tau$ пропорционален $P_{S} t_{p} / C$, в то время как сигнал $V_{S C}$ при постоянном облучении пропорционален $P_{S} / G_{0}=P_{S} \tau / C$. Следовательно, при увеличении теплопроводности болометра $G_{0}$ (и соответственно при понижении $\tau$ ) пиковое значение $V_{S I}$ практически не зависит от $G_{0}$ до тех пор, пока $\tau>t_{p}$. Это хорошо видно из эксперимента. Величина измеренного импульсного сигнала $V_{S I}$ при высоком вакууме лишь незначительно превышает величину сигнала при низком вакууме, в то время как в области постоянного освещения соответствующие сигналы $V_{S C}$ различаются в 20 раз, что соответствует увеличению $G_{0}$ (и уменьшению $\tau)$ в 20 раз. Столь сильное уменьшение $\tau$ видно также по зависимости величины сигнала $V_{S I}$ от времени. При высоком вакууме после окончания импульса излучения сигнал $V_{S I}$ уменьшается примерно в 1.5 раза за $9 \mathrm{~ms}$ (кривая 3 на рис. 4), в то время как при низком вакууме он практически полностью затухает за 2-3 ms (кривая 5).

На рис. 5 приведены экспериментальная и теоретические зависимости пикового значения сигнала ММБП от величины $\tau$ при его облучении импульсами ИК излучения. Время тепловой релаксации болометров уменьшалось по мере натекания газа в вакуумный корпус ММБП

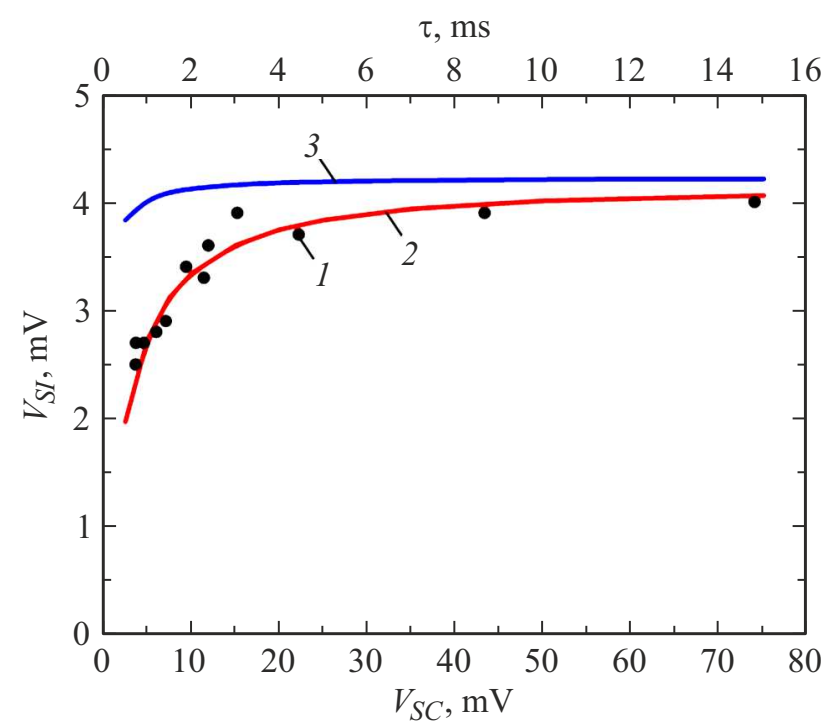

Рис. 5. Экспериментальная (1) и теоретические $(2,3)$ зависимости пикового значения сигнала ММБП $V_{S I}$ от времени тепловой релаксации $\tau$ (верхняя шкала), вычисляемого через значения сигнала $V_{S C}$ в области постоянной засветки (нижняя шкала), при облучении импульсами ИК излучения длительностью $1(1,2)$ и $0.1 \mathrm{~ms}(3)$. 

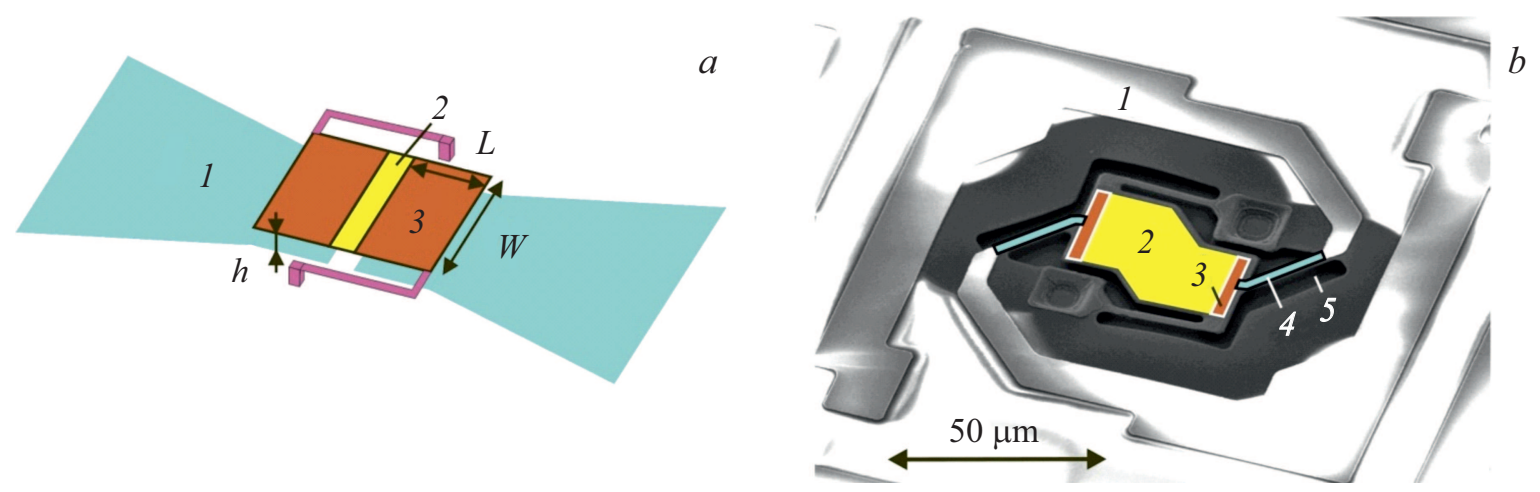

Рис. 6. Болометры антенного типа с емкостной $(a)$ и резистивной связью $(b) .1$ - антенна, $2-$ нагрузка (нагреватель), 3 - низкоомный контакт к нагрузке и обкладка конденсатора емкостной связи, 4 - шина резистивной связи антенны с нагревателем, 5 - область вытравленного жертвенного слоя.

и определялось по соотношению $\tau=\tau 0\left(V_{S C} / V_{S C 0}\right)$; $V_{S C 0}$ и $V_{S C}$ - значения сигнала в области постоянной засветки (строки 220-240) при высоком и пониженном вакууме соответственно. Теоретические зависимости пикового значения сигнала $V_{S I}$ от величины $\tau$ рассчитывали по интегральным соотношениям (5)-(7), в которых использовалась форма импульса излучения, экспериментально определенная путем измерения величины сигналов ММБП при медленном вращении диска. Хорошее соответствие экспериментальной и рассчитанной зависимости для импульса излучения длительностью $1 \mathrm{~ms}$ получили, приняв теплоемкость болометра $C$ равной $1.2 \cdot 10^{-9} \mathrm{~J} / \mathrm{K}$ (при этом $G_{0}=0.8 \cdot 10^{-7} \mathrm{~W} / \mathrm{K}$ ), что соответствует характерным значениям для используемого в эксперименте ММБП с размером пикселя $51 \mu \mathrm{m}$ [25]. В соответствии с изложенным выше величина сигнала слабо изменяется до тех пор, пока время тепловой релаксации заметно больше длительности импульса. Эта особенность позволяет разрабатывать приемники коротких THz-импульсов излучения, основанные на применении болометров с малыми значениями времени тепловой релаксации, что, в свою очередь, позволяет повысить кадровую частоту и открывает путь к использованию болометров с резистивно-связанной, термически неизолированной антенной, обладающих повышенной теплопроводностью и, следовательно, пониженной чувствительностью к постоянному излучению.

\section{3. Неохлаждаемые микроболометрические приемники импульсного THz-излучения с резистивно-связанными антеннами}

При разработке болометров с антеннами важно обеспечить эффективное сопряжение антенны и резистивной нагрузки (нагревателя), в которой выделяется энергия падающей на болометр электромагнитной волны. В THzдиапазоне частот успешно применяют два способа сопряжения. В первом, антенна располагается непосредственно на термоизолированной мембране вместе с нагрузкой. В этом случае их связь осуществляется прямым, резистивным способом. Во втором, антенна располагается на подложке, над которой подвешен болометр, а связь с нагрузкой, расположенной на термоизолированной мембране болометра, выполняется с помощью емкостной связи через тонкий вакуумный зазор (рис. 6, a). Обычно применяют микроболометры, сочетающие оба варианта $[8,9]$. Такие конструкции сложно применить для регистрации излучения с частотой менее $1 \mathrm{THz}$, поскольку низкая частота излучения затрудняет использование емкостной связи, а большие размеры антенны не позволяют поместить ее на маленькой (примерно $25 \times 25 \mu \mathrm{m})$ мембране болометра или, в противном случае, значительно повысят теплоемкость болометра. Действительно, импеданс $Z_{C}$ емкостной связи между антенной и нагрузкой можно оценить величиной $2 /\left(C_{e} \omega\right)$, соответствующей двум включенным последовательно с нагрузкой емкостям $C_{e}$, образованным электродами размером $W \times L \approx 25 \times 10 \mu \mathrm{m}$, разделенными вакуумным зазором $h \approx 2 \mu \mathrm{m}$ (рис. 6,a). На частотах $\omega / 2 \pi \approx 0.3 \mathrm{THz}$ импеданс $Z_{C} \approx 960 \Omega$, что значительно превышает импеданс вакуума, равный $377 \Omega$, и затрудняет согласование антенны и нагрузки, особенно в случае разработки широкополосного приемника.

Для приемников импульсного THz-излучения можно применить резистивную связь между антенной, расположенной на подложке (например, кремниевой, в которой обычно выполняется схема считывания сигналов мультиплексор), и нагрузкой, расположенной на мембране болометра (рис. 6, $b$ ). В этом случае нагрузка занимает практически всю поверхность мембраны, что способствует быстрому и однородному нагреванию тонкой мембраны при поглощении импульса излучения. Тепло распространяется поперек мембраны и характерное время прогревания $\tau_{d}$ мембраны из нитрида кремния толщиной $d=0.3 \mu \mathrm{m}$ составляет $\sim c \rho d^{2} / \kappa \approx 7 \cdot 10^{-8} \mathrm{~s}$, 
где $\rho, c$ и $\kappa-$ плотность, удельные теплоемкость и теплопроводность нитрида кремния, равные $2.2 \mathrm{~g} / \mathrm{cm}^{3}$, $1.5 \mathrm{~J} / \mathrm{gK}$ и $0.045 \mathrm{~W} / \mathrm{cmK}$ соответственно [26]. Наличие низкоомных контактов к резистивной нагрузке (рис. $6, b$ ), в которых практически не выделяется тепло, приведет к неоднородности выделения тепла и, как следствие, к перераспределению тепла вдоль мембраны. Однако при малой ширине а таких контактов $(a \approx 1 \mu \mathrm{m})$ и расположению их перпендикулярно контактам к термочувствительному слою, или при последовательном чередовании полос контактов и резистивной нагрузки (шириной $a \approx 1 \mu \mathrm{m}$ каждая), влияние продольного перераспределения тепла на величину сигнала может быть сведено к минимуму, а характерное время прогрева мембраны и соответственно установления сигнала может быть оценено величиной $\sim c \rho a^{2} / \kappa \approx 7 \cdot 10^{-7} \mathrm{~s}$. Остывание болометра после окончания импульса излучения преимущественно происходит вследствие оттока тепла от мембраны по тонким несущим балкам, обладающим малой теплопроводностью. Время остывания или время тепловой релаксации болометра $\tau=C / G$ для типичных болометров составляет $\sim 10 \mathrm{~ms}$ [26], многократно превышая время прогревания мембраны $\tau_{d}$, и, следовательно, определяет его быстродействие: величина температурного отклика болометра на измеряемый импульс излучения будет слабо зависеть от предыдущего импульса излучения, если между ними будет интервал времени, больше или порядка $3 \tau$.

На рис. $6, b$ приведено электронно-микроскопическое изображение тестового микроболометра, сопряженного с квазиспиральной антенной и изготовленного на мультиплексоре с размером пикселя $51 \times 51 \mu \mathrm{m}$. Антенна занимает площадь $3 \times 3$ пикселя, а микроболометр подключен к мультиплексору в центральном пикселе каждого массива $3 \times 3$ [18]. При этом следует отметить, что с целью повышения чувствительности и быстродействия болометра выгоднее применять микроболометры меньших размеров, например, $25 \times 25 \mu \mathrm{m}$, обладающие, по сравнению с микроболометрами размером $51 \times 51 \mu \mathrm{m}$ примерно в 4 раза меньшей теплоемкостью. Это обусловлено тем, что величина отклика болометра на короткое $\left(t_{p e} \ll \tau\right)$ импульсное излучение $T\left(t_{2}\right)-T\left(t_{1}\right) \approx Q_{S} / C$ обратно пропорциональна его теплоемкости, а время остывания болометра $\tau=C / G$ пропорционально теплоемкости. В данной конструкции антенна расположена на жертвенном слое, толщина которого может составлять $\sim 2-5 \mu \mathrm{m}$. С целью оптимизации эффективности антенны аналогично работам [7-9] между жертвенным слоем и кремниевой подложкой может быть нанесен толстый слой диэлектрика (например, оксид или нитрид кремния толщиной $\sim 10 \mu \mathrm{m}$ или больше). Принимая сопротивления нагрузки и каждой шины резистивной связи $r$ соответственно равными 100 и $15 \Omega$, найдем, что доля выделяющегося в нагрузке джоулевого тепла при поглощении импульса излучения составляет 70\%. При этом, согласно закону Франца-Видемана, теплопроводность шин резистивной связи, обусловленная носителями заряда, $G_{e}=2 L T_{C} / r$, составит $1.0 \cdot 10^{-6} \mathrm{~W} / \mathrm{K}$. Здесь $L-$ постоянная Франца-Видемана, равная $2.45 \cdot 10^{-8} \mathrm{~W} \Omega / \mathrm{K}^{2}$, и $T_{C}$ - температура подложки, которую мы принимаем равной $300 \mathrm{~K}$. Полная теплопроводность болометра $G$ будет немного выше из-за неучтенных здесь, во-первых, теплопроводности решетки металла, которая для металлов с высокой электропроводимостью (таких как $\mathrm{Au}, \mathrm{Cu}$, $\mathrm{Ag}, \mathrm{Al})$ может быть малой по сравнению с электронной теплопроводностью [27,28], и, во-вторых, теплопроводности по несущим балкам с металлическими шинами, соединяющими термочувствительный элемент болометра со схемой считывания сигнала, типичное значение которой менее $1.0 \cdot 10^{-7} \mathrm{~W} / \mathrm{K}$ [29-31]. В результате, учитывая, что величина теплоемкости мембраны $C$ для микроболометров размером 25-28 $\mu \mathrm{m}$ может составлять $\sim 4 \cdot 10^{-10} \mathrm{~J} / \mathrm{K}[29-31]$, найдем тепловое время релаксации болометра $\tau=C / G \approx 0.4 \mathrm{~ms}$ и, следовательно, такой болометр способен измерять энергию каждого из импульсов излучения, следующих друг за другом с частотой $\sim 1 / 3 \tau \approx 830 \mathrm{~Hz}$.

Проводя измерение сигнала болометра (сразу после прихода импульса излучения) путем подачи импульсного напряжения смещения болометра $V_{D} \approx 2 \mathrm{~V}$ в течение времени интегрирования сигнала $\tau_{i} \approx 30 \mu \mathrm{s}$, и приравнивая величину сигнала $V_{S I}=\alpha\left(0.7 Q_{S} / C\right) V_{D} \approx$ $\approx \alpha\left(0.7 \eta Q_{S I} / C\right) V_{D}$ к полному шуму схемы измерения (приведенному к болометру), который обычно составляет $\sim 10 \mu \mathrm{V}[32,33]$, можно оценить минимальную разрешимую энергию падающего на болометр импульса излучения $Q_{S I} \approx 3 \cdot 10^{-13} \mathrm{~J}$. Здесь $\eta-$ коэффициент

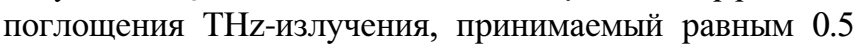
(свыше 80\% падающего излучения поглощается антенной $[8,9]$ и 70\% этой энергии выделяется в резистивной нагрузке) и $\alpha-$ ТКС болометра, который, как и ранее, принимаем равным $-2 \% / \mathrm{K}$ [2]. Для сравнения укажем, что средние мощности излучения фотопроводящей антенны и THz-квантово-каскадного лазера составляют примерно $0.1 \mathrm{~mW}$ [13] и $10 \mathrm{~mW}$ [34]. Энергии одного импульса длительностью $100 \mu \mathrm{s}$ соответственно составят $10^{-8}$ и $10^{-6} \mathrm{~J}$, что позволит в режиме snapshot

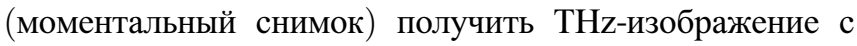
отношением сигнал/шум $\approx 40$ на матрицах форматом $32 \times 24$ и $320 \times 240$ соответственно.

Для обеспечения режима snapshot может быть использована конструкция мультиплексора с прямой инжекцией, аналогичная применяемым в работах $[35,36]$, в которой в каждой ячейке фоточувствительной матрицы производится вычитание „темнового“ тока с последующим интегрированием разницы на накопительной емкости. Поскольку в отличие от работ [35,36] в нашем случае измерение нужно произвести за время меньшее, чем $\tau \approx 0.4 \mathrm{~ms}$, например за $30 \mu \mathrm{s}$, для обеспечения среднеквадратичного шума Джонсона менее $10 \mu \mathrm{V}$, необходимо использовать болометры с сопротивлением менее $300 \mathrm{k} \Omega$. При условии обеспечения 
вычитания не менее 95\% „темнового“ тока, для выполнения интегрирования сигнала болометров с сопротивлением $100-300 \mathrm{k} \Omega$ достаточно накопительной емкости $C_{i} \approx 3-10 \mathrm{pF}$, заряжаемой не менее, чем до $3 \mathrm{~V}$. Такая величина емкости может быть реализована в мультиплексоре при использовании полевого оксида кремния толщиной $15 \mathrm{~nm}$. Площадь конденсатора составит не более $37 \times 37-75 \times 75 \mu \mathrm{m}$, что вполне реально для приемников терагерцового излучения, поскольку в этом случае размер пикселя, определяемый размером антенны, может составлять $100 \times 100 \mu \mathrm{m}$ и более. Компенсирующий „темновой“ ток может быть задан путем применения либо опорных болометров (нечувствительных к измеряемому излучению, например, выполненных без антенны) [37], либо токовых зеркал [38], размещенных в каждой ячейке ММБП. Отметим также, что для матричного приемника форматом $160 \times 120$ при частоте кадров $1000 \mathrm{~Hz}$ тактовая частота выходного сигнала составит $20 \mathrm{MHz}$ (или $5 \mathrm{MHz}$ при наличии 4 каналов вывода), что не создаст схемотехнических проблем.

\section{Заключение}

Исследована зависимость сигнала болометра от длительности, формы и периода следования импульсов излучения, а также от времени тепловой релаксации болометра. Получены аналитические соотношения для температурного отклика болометров на периодические импульсы излучения трех различных форм: прямоугольной, треугольной и гауссовой. Теоретически показано и на примере инфракрасных болометров экспериментально подтверждено, что температурный отклик определяется теплоемкостью болометра и слабо зависит от его теплопроводности при условии, что длительность импульсов излучения значительно меньше периода их следования и времени тепловой релаксации болометра. Эта особенность позволяет при регистрации коротких ИК и THz-импульсов применять конструкции болометров, характеризующиеся повышенной теплопроводностью и, следовательно, пониженным временем тепловой релаксации, что делает их быстродействующими, практически не понижая чувствительность. В частности, показано, что микроболометры с резистивно-связанной, термически неизолированной антенной, обладающие повышенной теплопроводностью и пониженной чувствительностью к излучению постоянной интенсивности, могут быть высокоэффективными при регистрации THzимпульсного излучения. На основе таких болометров могут быть разработаны неохлаждаемые матричные приемники импульсного THz-излучения, характеризующиеся минимальной обнаружимой энергией менее $1 \cdot 10^{-12} \mathrm{~J}$ и кадровой частотой до $1000 \mathrm{~Hz}$. Рассмотренный выше подход также может быть применен к болометрическим приемникам миллиметрового излучения.

\section{Финансирование работы}

Исследование выполнено в рамках Государственного задания № 0242-2021-0007.

\section{Конфликт интересов}

Авторы заявляют, что у них нет конфликта интересов.

\section{Приложение}

В общем случае, когда эффективная длительность импульса излучения $t_{p e}$ может быть сравнима со временем тепловой релаксации $\tau$, величины $D T\left(t_{1}, t\right)$ и $D T\left(t_{1}, t_{2}\right)$, входящие в выражения (5)-(7), для трех типов импульсов, заданных соотношениями (8) и изображенных на рис. 1, могут быть выражены в аналитическом виде:

1) для прямоугольного импульса:

$$
\begin{gathered}
D T_{1}\left(t_{1}, t\right)=\frac{Q_{S}}{C} \frac{1}{\left(t_{p} / \tau\right)}\left[\exp (t / \tau)-\exp \left(-t_{p} / 2 \tau\right)\right] \\
\text { при } t_{1}<t<t_{2} \\
D T_{1}\left(t_{1}, t_{2}\right)=\frac{Q_{S}}{C} \frac{\operatorname{sh}\left(t_{p} / 2 \tau\right)}{t_{p} / 2 \tau}
\end{gathered}
$$

где $t_{1}=-t_{p} / 2, t_{2}=t_{p} / 2$;

2) для треугольного импульса:

$$
\begin{gathered}
D T_{2}\left(t_{1}, t\right)=\frac{Q_{S}}{C} \frac{1}{\left(t_{p} / 2 \tau\right)^{2}}\left[\exp \left(-\frac{t_{p}}{2 \tau}\right)-\exp \left(\frac{t}{\tau}\right)\right. \\
\left.+\left(\frac{t_{p}}{2 \tau}+\frac{t}{\tau}\right) \exp \left(\frac{t}{\tau}\right)\right], \quad \text { при } t_{1}<t<0, \\
D T_{2}\left(t_{1}, t\right)=\frac{Q_{S}}{C} \frac{1}{\left(t_{p} / 2 \tau\right)^{2}}\left[\exp \left(-\frac{t_{p}}{2 \tau}\right)+\exp \left(\frac{t}{\tau}\right)\right. \\
\left.-2+\left(\frac{t_{p}}{2 \tau}-\frac{t}{\tau}\right) \exp \left(\frac{t}{\tau}\right)\right], \text { при } 0<t<t_{2}, \\
D T_{2}\left(t_{1}, t_{2}\right)=\frac{Q_{S}}{C} 2 \frac{\operatorname{ch}\left(t_{p} / 2 \tau\right)-1}{\left(t_{p} / 2 \tau\right)^{2}} .
\end{gathered}
$$

где $t_{1}=-t_{p} / 2, t_{2}=t_{p} / 2$;

3) для гауссова импульса:

$$
\begin{gathered}
D T_{3}\left(t_{1}, t\right)=\frac{Q_{S}}{C} \frac{1}{2}\left\{1+\operatorname{erf}\left[\frac{1}{\sqrt{2}}\left(\frac{t}{t_{p} / b}-\frac{t_{p} / b}{\tau}\right)\right]\right\} \\
\times \exp \left[\frac{\left(t_{p} / b\right)^{2}}{2 \tau^{2}}\right], \text { при } t_{1}<t<t_{2}, \\
D T_{3}\left(t_{1}, t_{2}\right)=\frac{Q_{S}}{C} \exp \left[\frac{\left(t_{p} / b\right)^{2}}{2 \tau^{2}}\right],
\end{gathered}
$$

где $t_{1}=-n\left(t_{p} / 2\right), t_{2}=n\left(t_{p} / 2\right), n \geq 1$ и $\operatorname{erf}(x)-$ функция ошибок.

Отметим, что приведенные на рис. 2 зависимости, рассчитанные с использованием интегрального (7) и аналитических (П1)-(П3) соотношений при $n=1.5$, 
совпадают с точностью до ширины линии. При этом следует подчеркнуть, что соотношения (П1) и (П2) являются точными, а (П3) - приближенным. Точность последнего возрастает с ростом параметра $n$. При $n=1$ и 1.5 относительная мощность излучения $P_{S}\left(t_{1}\right) / P_{S}(0)=P_{S}\left(t_{2}\right) / P_{S}(0)$ на краях интервала $\left(t_{1}, t_{2}\right)$ составляет $4.3 \cdot 10^{-2}$ и $8.5 \cdot 10^{-4}$ соответственно.

\section{Список литературы}

[1] A. Rogalski. Progress in Quantum Electronics, 36 (2-3), 342 (2012). DOI: 10.1016/j.pquantelec.2012.07.001

[2] F. Niklaus, C. Vieider, H. Jakobsen. Proc. SPIE, 6836, 68360D (2007). DOI: $10.1117 / 12.755128$

[3] A. Rogalski. Opto-Electron. Rev., 21 (4), 406 (2013). DOI: $10.2478 / \mathrm{s} 11772-013-0110-\mathrm{x}$

[4] A.W.M. Lee, B.S. Williams, S. Kumar, Q. Hu, J.L. Reno. IEEE Photonics Technol. Lett., 18 (13), 1415 (2006). DOI: 10.1109/LPT.2006.877220

[5] N. Oda. C.R. Physique, 11 (7-8), 496 (2010). DOI: $10.1016 /$ j.crhy.2010.05.001

[6] M.A. Dem'yanenko, D.G. Esaev, B.A. Knyazev, G.N. Kulipanov, N.A. Vinokurov. Appl. Phys. Lett., 92 (13), 131116 (2008). DOI: 10.1063/1.2898138

[7] N. Nemoto, N. Kanda, R. Imai, K. Konishi, M. Miyoshi, S. Kurashina, T. Sasaki, N. Oda, M. Kuwata-Gonokami. IEEE Trans. on Terahertz Sci. Technol., 6(2), 175 (2016). DOI: 10.1109/TTHZ.2015.2508010

[8] F. Simoens, J. Meilhan. Philosophical Transactions of the Royal Society of London A: Mathematical, Physical and Engineering Sciences, 372 (2012), 20130111 (2014). DOI: 10.1098/rsta.2013.0111

[9] F. Simoens, J. Meilhan, J.-A. Nicolas. J. Infrared Milli Terahz Waves, 36, 961 (2015). DOI: 10.1007/s10762-015-0197-x

[10] G.S. Kent, B.R. Clemesha, R.W. Wright. J. Atmospheric Terrestrial Phys., 29 (2), 169 (1967). DOI: 10.1016/0021-9169(67)90131-6

[11] G.S. Kent, R.W. Wright. J. Atmospheric Terrestrial Phys., 32 (5), 917 (1970). DOI: 10.1016/0021-9169(70)90036-X

[12] C.W. Berry, M.R. Hashemi, M. Jarrahi. Appl. Phys. Lett., 104 (8), 081122 (2014). DOI: 10.1063/1.4866807

[13] D.S. Kim, D.S. Citrin. Appl. Phys. Lett., 88(16), 161117 (2006). DOI: $10.1063 / 1.2196480$

[14] H. Hirori, A. Doi, F. Blanchard, K. Tanaka. Appl. Phys. Lett., 98 (8), 091106 (2011). DOI: 10.1063/1.3560062

[15] M.A. Belkin, F. Capasso. Phys. Scr., 90 (11), 118002 (2015). DOI: $10.1088 / 0031-8949 / 90 / 11 / 118002$

[16] L. Li, L. Chen, J. Zhu, J. Freeman, P. Dean, A. Valavanis, A.G. Davies, E.H. Linfield. Electron. Lett., 50 (4), 309 (2014). DOI: $10.1049 / \mathrm{el} .2013 .4035$

[17] Q. Lu, M. Razeghi. Photonics, 3 (3), 42 (2016). DOI: $10.3390 /$ photonics3030042

[18] М.А. Демьяненко, Д.Г. Есаев, В.Н. Овсюк, Б.И. Фомин, А.Л. Асеев, Б.А. Князев, Г.Н. Кулипанов, Н.А. Винокуров. Оптич. журн., 76 (12), 5 (2009). [M.A. Dem'yanenko, D.G. Esaev, V.N. Ovsyuk, B.I. Fomin, A.L. Aseev, B.A. Knyazev, G.N. Kulipanov, N.A. Vinokurov. J. Opt. Technol., 76 (12), 739 (2009). DOI: 10.1364/JOT.76.000739]

[19] D. Dufour, L. Marchese, M. Terroux, H. Oulachgar, F. Généreux, M. Doucet, L. Mercier, B. Tremblay, C. Alain, P. Beaupré, N. Blanchard, M. Bolduc, C. Chevalier,
D.D’Amato, Y. Desroches, F. Duchesne, L. Gagnon, S. Ilias, H. Jerominek, F. Lagacé, J. Lambert, F. Lamontagne, L.L. Noc, A. Martel, O. Pancrati, J.-E. Paultre, T. Pope, F. Provençal, P. Topart, C. Vachon, S. Verreault, A. Bergeron. J. Infrared Milli Terahz Waves, 36(10), 922 (2015). DOI: $10.1007 / \mathrm{s} 10762-015-0181-5$

[20] S. Zwerdling, R.A. Smith, J.P. Theriault. Infrared Phys., 8 (4), 271 (1968). DOI: 10.1016/0020-0891(68)90036-5

[21] Handbook of Optics. Vol. I. Fundamentals, Techniques and Design, ed. by M. Bass, E.W. Van Stryland, D.R. Williams, W.L. Wolfe. - 2nd ed. (McGraw-Hill, NY.-San Francisco-Washington, D.C. - etc, 1995).

[22] В.Ф. Зайцев, А.Д. Полянин. Справочник по обыкновенным дифберенциальным уравнениям (Физматлит, М., 2001) [A.D. Polyanin, V.F. Zaitsev. Handbook of Exact Solutions for Ordinary Differential Equations (Chapman \& Hall/CRC, Boca Raton-London-NY.-Washington, D.C. 2003)]

[23] М.А. Демьяненко, А.Ф. Кравченко, В.Н. Овсюк. Автометрия, 41 (5), 108 (2005).

[24] В.Ш. Алиев, М.А. Демьяненко, Д.Г. Есаев, И.В. Марчишин, В.Н. Овсюк, Б.И. Фомин. Успехи прикладной физики, 1 (4), 471 (2013).

[25] М.А. Демьяненко, Б.И. Фомин, Л.Л. Васильева, С.А. Волков, И.В. Марчишин, Д.Г. Есаев, В.Н. Овсюк, В.Л. Дшхунян, Е.Б. Володин, А.В. Ермолов, П.П. Усов, В.П. Чесноков, Ю.С. Четверов, П.Н. Кудрявцев, А.Е. Здобников, А.А. Игнатов. Прикладная физика, 4, 124 (2010).

[26] P. Eriksson, J.Y. Andersson, G. Stemme. J. Microelectromechanical Systems, 6 (1), 55 (1997).

[27] C. Kittel. Introduction to Solid State Physics, 8th Edition. (John Wiley and Sons, NY., 2005)

[28] F.H. Schofield. Proceed. Royal Society A: Mathematical, Phys. Engineer. Sci., 107 (742), 206 (1925). DOI: $10.1098 /$ rspa.1925.0016

[29] F. Niklaus, A. Decharat, C. Jansson, G. Stemme. Infrared Phys. Technol., 51 (3), 168 (2008). DOI: $10.1016 /$ j.infrared.2007.08.001

[30] M. Kohin, N. Butler. Proc. SPIE, 5406, 447 (2004). DOI: $10.1117 / 12.542482$

[31] J.J. Yon, A. Astier, S. Bisotto, G. Chamingís, A. Durand, J.L. Martin, E. Mottin, J.L. Ouvrier-Buffet, J.L. Tissot. Proc. SPIE, 5783, 432 (2005). DOI: 10.1117/12.606487

[32] D. Svard, C. Jansson, A, Alvandpour. Analog Integr Circ Sig Process, 77 (1), 29 (2013). DOI: 10.1007/s10470-013-0116-9

[33] A. Tanaka, K. Chiba, T. Endoh, K. Okuyama, A. Kawahara, K. Iida, N. Tsukamoto. Proc. SPIE, 4130, 160 (2000). DOI: $10.1117 / 12.409858$

[34] B.S. Williams. Nature Photon., 1 (9), 517 (2007). DOI: $10.1038 /$ nphoton.2007.166

[35] C.H. Hwang, C.B. Kim, Y.S. Lee, H.C. Lee. Electron. Lett., 44 (12), 732 (2008). DOI: 10.1049/el:20080879

[36] S. Ajmera, J. Brady, C. Hanson, T. Schimert, A.J. Syllaios, M. Taylor. Proc. SPIE, 8012, 80121L (2011). DOI: $10.1117 / 12.884249$

[37] J.L. Tissot, C. Trouilleau, B. Fieque, A. Crastes, O. Legras. Opto-Electron. Rev., 14 (1), 25 (2006). DOI: $10.2478 / \mathrm{s} 11772-006-0004-2$

[38] F. Simoens, M. Tchagaspanian, A. Arnaud, P. Imperinetti, G. Chammings, J.J. Yon, J.L. Tissot. Proc. SPIE, 6542, 65421T (2007). DOI: $10.1117 / 12.719310$ 